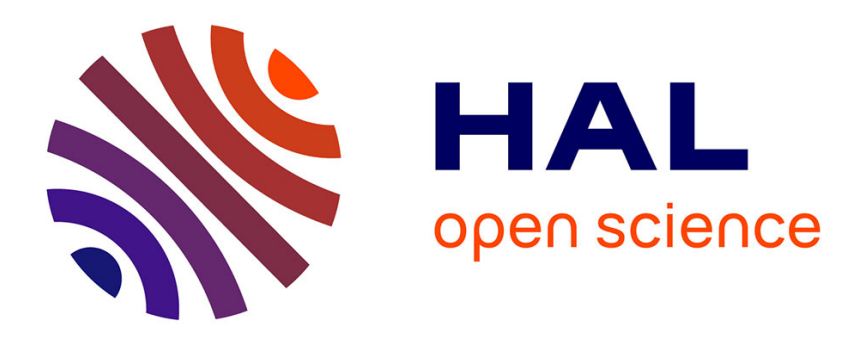

\title{
L'entreprise à mission et ses partenaires
}

Armand Hatchuel

\section{To cite this version:}

Armand Hatchuel. L'entreprise à mission et ses partenaires: Propos recueillis par Anne Querrien. Multitudes, 2018, 2018/3 (72), pp.211-217. 10.3917/mult.072.0211 . hal-01895375

\section{HAL Id: hal-01895375 \\ https://hal-mines-paristech.archives-ouvertes.fr/hal-01895375}

Submitted on 6 Nov 2018

HAL is a multi-disciplinary open access archive for the deposit and dissemination of scientific research documents, whether they are published or not. The documents may come from teaching and research institutions in France or abroad, or from public or private research centers.
L'archive ouverte pluridisciplinaire HAL, est destinée au dépôt et à la diffusion de documents scientifiques de niveau recherche, publiés ou non, émanant des établissements d'enseignement et de recherche français ou étrangers, des laboratoires publics ou privés. 
Armand Hatchuel. L'entreprise à mission et ses partenaires :

Propos recueillis par Anne Querrien.

Multitudes, Éd. Inculte, 2018, 2018/3 (72), pp.211-217.

Multitudes: Comment en êtes-vous venu à proposer que les entreprises se dotent de missions explicites?

Armand Hatchuel : Nous sommes partis de la crise financière de 2008, et de ce qui nous a semblé une erreur profonde d'interprétation. Pour nous il ne s'agissait par de la même crise du capitalisme qu'en 1929, épisode qui lui-même répéterait à l'identique les crises précédentes. Nous, formateurs d'ingénieurs, dont certains sont appelés à devenir chefs d'entreprise ${ }^{1}$, n'avions pas à nous identifier à des médecins soignant un parasite qui donnerait de temps en temps de la fièvre et avec lequel il faudrait se résigner à vivre.

L'idée que le capitalisme serait un invariant anhistorique, condamné à un même type de crise récurrente ou en voie d'effondrement, est intenable scientifiquement. Cette épistémologie est fausse. Pour nous ce qui est fondamental dans le capitalisme moderne qui émerge à la fin du XIX siècle c'est d'une part l'incorporation à l'activité productive des découvertes scientifiques et d'autre part le développement du mouvement ouvrier. En 1929 on était encore dans les débuts de l'industrie scientifique, dans les débuts du mouvement ouvrier, dans les débuts de l'entreprise, dans sa forme moderne. La crise frappe un tissu d'entreprises moyennes et petites avec des moyens d'information limités, sans experts, sans cols blancs, sans journaux spécialisés, sans moyens de comprendre l'évolution en cours.

En 2005, date du début de la crise des subprimes, la situation est bien différente. Des entreprises géantes dominent le monde, et le tissu économique par la sous-traitance. Et surtout, sept ou huit grandes banques forment un oligopole mondial, et jouent un rôle essentiel dans tout le système financier, y compris les fonds souverains.

La crise qui éclate en 2008 est donc une crise du management de ces grandes puissances économiques, une crise de leur gouvernance.

Les enquêtes confirment en effet que les banques "n'étaient plus des banques", soucieuses d'éviter les risques et de sauvegarder leurs réserves. Elles étaient au contraire à la recherche active profits élevés à de court terme et prêtes pour cela a s'engager dans des produits financiers au risque élevé et non assuré. La chute de Lehman Brothers ${ }^{2}$ n'était que le point d'orgue d'une spéculation sur l'accession à la propriété des personnes à faible revenu, qui avait dépassé toutes les bornes que se mettait traditionnellement l'industrie bancaire.

$\mathrm{Au}$ fond, ce qui s'est passé dans les banques à ce moment-là ressemble à ce qui a fait chuter Enron ${ }^{3}$, Parmalat 4 , Worldcom ${ }^{5}$, quelques années avant: des comportements

\footnotetext{
${ }^{1}$ Hatchuel A. et Segrestin B., Refonder l'entreprise, Le Seuil/La République des idées, 2012.

2 https://en.wikipedia.org/wiki/Bankruptcy_of_Lehman_Brothers

${ }^{3}$ https://www.lesechos.fr/16/10/2002/LesEchos/18762-006-ECH_enron--oucomment-le-modele-est-devenu-un-scandale-planetaire.htm

${ }^{4}$ Cf. Ignacio Ramonet, « Le scandale Parmalat», Le Monde diplomatique, février 2004.
} 
complètements irrationnels, et parfois illégaux, destinés à garantir des profits élevés aux actionnaires, et souvent dans une confusion complète entre l'industrie et la finance. Ces comportements sont loin d'avoir disparu, il y a seulement quelques mois, le géant britannique du BTP, Carillion, détenteur de nombreux contrats public-privé pour la gestion des cantines scolaires, des prisons et d'autres services publics, s'est également déclaré en faillite en janvier 2018, après avoir versés pendant des années des dividendes qui avaient fait croire à sa bonne santé6.

Nous estimons que cette série de crises ne relèvent pas d'explications anhistoriques sur les contradictions internes du capitalisme. Il ne faut pas analyser les faits « économiques » comme s'ils relevaient de lois identiques lorsqu'ils relèvent de régimes d'activité et de régimes de responsabilité complètement différents. Par exemple, l'incorporation de la recherche scientifique dans l'activité industrielle reste à nos yeux une transformation de civilisation, d'ordre anthropologique, dont l'analyse a été pour l'instant négligée.

Ce qui s'est passé pendant ces dernières décennies doit être expliqué, par une donnée de la période : de nouvelles règles de gouvernance des grandes entreprises concernées, et par l'incapacité de ces règles à empêcher des dérives de direction de l'entreprise allant jusqu'à mettre cette dernière en péril.

La règle de gouvernance à l'origine de ces conduites dévoyées, ce n'est pas la maximisation du profit, mais la maximisation de la valeur pour l'actionnaire qui conduit ces entreprises à devoir distribuer des dividendes ou à défendre le cours de l'action en limitant l'investissement ou en accumulant les dettes, voire à créer du déficit masqué. La stratégie qui consiste à sortir de la mission productrice de l'entreprise pour spéculer sur les marchés financiers est une stratégie erronée. William Lazonick a expliqué dans la Harvard Business Review ${ }^{7}$ que dans les années 2000-2008 les bénéfices avaient été utilisés à 90\% pour racheter des actions et faire monter leur cours artificiellement, ce qui a appauvri les Américains : il a appelé cela le paradoxe du " profit sans prospérité ». Jusqu'aux années 1970, les grandes entreprises étaient vues comme les fleurons de la nation. On se souvient de l'adage : ce qui est bon pour General Motors est bon pour les Etats Unis. Et on aurait dit sans hésiter la même chose de Renault pour la France. Cette représentation collective s'est brisée, car les mécanismes qui faisaient tendre la grande entreprise vers l'accomplissement du bien collectif ont disparu.

Multitudes: Comment s'est transformée la gouvernance des entreprises ces dernières années?

Armand Hatchuel : Ce qui s'est transformé d'abord c'est l'actionnariat. On a connu une mutation mondiale de la détention des actions sur trente ans. En deux décennies la durée de détention moyenne des actions est passée de 5 à 6 ans à 6 mois, et la majorité des titres sont détenues par de grands fonds ou des entreprises. Les mécanismes d'achat

\footnotetext{
${ }^{5}$ Cf. https://www.lesechos.fr/16/03/2005/LesEchos/19372-071-ECH_worldcom--retour-sur-un-scandale.htm

${ }^{6}$ https://www.lemoniteur.fr/article/btp-pourquoi-le-geant-britannique-carillion-sedeclare-en-faillite-35252671

7 https://www.ineteconomics.org/uploads/papers/LAZONICK_William_Profits-withoutProsperity-20140406.pdf
} 
et de vente ont été accéléré via internet, d'où une grande instabilité permanente de la détention des actions. Il en résulte une situation paradoxale : d'un côté l'actionnaire, en tant que "propriétaire » supposé de l'entreprise, n'a jamais été aussi puissant, pour imposer des codes de gouvernance en sa faveur, et des normes de rentabilité exclusivement centrées sur ses intérêts, mais d'un autre côté l'actionnaire est complètement découplé de la production réelle. C'est ce qui permet à des actionnaires " activistes » de révolutionner l'entreprise avec seulement 1\% du capital, en occupant l'espace des assemblées générales.

Ces actionnaires, puissants symboliquement, mobilisent les règles et les normes d'évaluation de la corporate governance, une soft law, qui ne voit l'entreprise que comme un actif financier. Par ailleurs, des normes comptables ont-elles-mêmes évolué et s'est imposé au plan international une philosophie des comptes qui adopte le point de vue de l'analyse actionnariale. Il faut noter que plus les crises d'entreprises se multiplient et plus la solution consiste à augmenter le pouvoir des actionnaires : c'est le cas pour la rémunération des dirigeants que la France essaye de limiter. Mais on a pensé que si la décision revenait aux actionnaires cela limiterait les rémunérations excessives : ce qui est loin d'être la réalité.

Dans le même temps, l'entreprise, comme action collective créatrice de nouvelles richesses, notamment par la recherche scientifique adapté à la transformation de la matière ou de l'organisation du travail, a complètement disparu du paysage intellectuel et politique. Elle n'est pas mentionnée dans les codes de bonne gouvernance qui ne s'intéressent qu'à la société par actions, à la collectivité des actionnaires. Dans les livres de droit des affaires c'est pareil, on ne s'intéresse qu'aux actifs financiers et aux droits des sociétés. Le seul conflit pris en compte c'est uniquement celui entre capital et travail, un binarisme qui ne correspond pas à la réalité.

Plus fondamentalement, nous nous sommes aperçus que l'entreprise était un point aveugle des sciences sociales, une réalité collective non traitée tant par le droit, que par la sociologie ou l'économie. Ce fut d'ailleurs l'objet d'un colloque de Cerisy particulièrement instructif 8 .

\section{Multitudes : Comment parlait-on de l'entreprise auparavant?}

Armand Hatchuel :

Avec Blanche Ségrestin, on s'est aperçus que Michel Foucault était ici très utile pour comprendre que l'entreprise, qui n'est pas une catégorie définie dans le droit, est apparue en occident sous la forme d'un paradigme associant savoir et pouvoir. Ce paradigme apparu dans les années 1880 en même temps que la multiplication des écoles de commerce et des écoles d'ingénieurs, faisait des entreprises des organisations bénéfiques pour la collectivité, dès lors qu'elles associaient travailleurs, ingénieurs et actionnaires dans l'organisation de nouvelles productions et dans la conduite de projets innovants. La société par actions qui était depuis 1867 un cadre possible pour le développement d'une entreprise n'occupait pas le devant de la scène. En 1916, quand Henri Fayol publie son Traité d'administration industrielle et générale, considéré comme le premier ouvrage de management des grandes entreprises, il réduit le rôle des actionnaires à la nomination des directeurs.

Le droit des sociétés est d'une très grande ambiguïté quant aux relations entre actionnaires et dirigeants d'entreprise. Il est compatible avec un paradigme de

\footnotetext{
${ }^{8}$ https://editions.scienceshumaines.com/l-entreprise_fr-549.htm
} 
l'entreprise ancré dans la culture du progrès et l'admiration des merveilles de l'industrie, que les ingénieurs-directeurs ont la capacité de mettre en valeur, notamment dans les grandes expositions universelles. Le rapport sociétés par action/entreprise industrielle va être en faveur de ces dernières pendant environ quarante ans. Mais ce même droit des sociétés peut aussi être lu comme l'obligation pour le dirigeant de l'entreprise de servir exclusivement les actionnaires, et non pas les autres partenaires que sont les salariés, l'Etat ou la société civile. Or, c'est cette lecture qui l'a emporté dans la doxa internationale : la confusion de l'entreprise avec la société par actions qui la finance est devenue l'épistémologie obligatoire commune. Le tissu industriel étant fortement lié par les interdépendances entre grandes entreprises et sous-traitants, cette vision financière de l'économie s'est propagée presque partout et surtout dans l'enseignement et la formation.

\section{Multitudes : Quelles sont les conséquences de cette épistémologie?}

Armand Hatchuel :

Depuis les années 1990, dans cette nouvelle épistémologie, les dirigeants ont connu une explosion de leurs rémunérations et celles-ci les incitent à remplir d'abord leurs obligations financières légale (fiduciary duties): la théorie de la responsabilité l'emporte sur la théorie de l'activité productive. Comme on l'a vu avec les scandales évoqués plus haut, des dérives importantes sont apparues dans la gestion des entreprises, d'autant plus saluées au départ qu'elles faisaient augmenter le cours de l'action. Ces manipulations ont fait croire que les profits augmentaient avec les dividendes alors qu'en réalité il y avait stagnation de la valeur ajoutée et de la dynamique industrielle.

La conception de l'entreprise que nous avons développé dans nos travaux, notamment avec le collège des Bernardins, repart du paradigme apparu à la fin du XIX siècle, mais en prenant acte des contributions de toutes les parties. Chaque "associé » à l'entreprise, qu'il soit actionnaire, salarié, ingénieur, dirigeant, doit gérer la société comme ses propres affaires, comme la ressource qui lui permet de garder et de développer son potentiel d'action. L'entreprise c'est une solidarité organisée entre partenaires aux qualités différentes. Dans son principe, cette solidarité dépasse complètement le rapport capital/travail car elle implique l'usage de la science souvent produite par la puissance publique, la prise de brevets, l'action des ingénieurs, et donc plus généralement la création collective . Evidemment le rapport à la science n'est plus le même aujourd'hui qu'à la fin du XIX siècle : les « dégâts du progrès » exigent un pluralisme scientifique, une réflexion sur les menaces dont sont lourds ces investissements. Mais le paradigme de l'entreprise comme association de partenaires dans une même création collective n'en a que plus d'actualité. Notre paradigme central s'exprime ainsi : "Nous faisons des entreprises pour créer des mondes qui nous plaisent». Et les entreprises ont la responsabilité de créer le futur que nous désirons, au lieu de le détruire, ou de produire des mondes invivables au nom du profit des actionnaires. Et pour cela nous devons réhabiliter, en le réinterprétant bien sûr, le management scientifique, le mouvement des rationalisateurs de la troisième république, et les autres groupes qui, dans le contexte qui était le leur, ont mis la création collective au poste de commandement. ${ }^{9}$

${ }^{9}$ https://www.universalis.fr/encyclopedie/paul-desjardins/. 
Bien que complètement opposée à la doxa ambiante sur le pouvoir des actionnaires notre conception de l'entreprise comme dispositif de création collective a commencé à intéresser, d'autant que le droit libéral et l'économie classique qui l'accompagne sont aveugles à la création collective. C'est ainsi que nous avons été souvent sollicités pour développer la principale idée de notre livre de 2012 : l'entreprise est une idée moderne qui s'installe au tournant du 20è siècle mais dont les prémisses se trouvent au 18è siècle lorsqu'on commence à appliquer les sciences naissantes à la production pour le bénéfice de la société.

\section{Multitudes : Comment voyez-vous cette restauration de l'entreprise aujourd'hui ?}

\section{Armand Hatchuel :}

Il faut pour cela reconnaître la distinction entre société par actions et entreprise. L'entreprise rassemble de facto sur un projet de création commun; la société lie les associés de jure, selon la loi. L'actionnaire doit être re-responsabilisé sur l'objectif commun de l'entreprise, au lieu de ne s'intéresser qu'au seul montant de ses dividendes. Cette évolution passe d'abord par une modification du code civil qui entérine de nouvelles responsabilités de la société anonyme. On doit affirmer que la société par actions est destinée à créer, et à accompagner, une entreprise commune, qui doit être gérée en prenant en compte les intérêt des parties "constituantes » ${ }^{10}$ (c'est le mot qu'ont choisi les syndicats et que retient le Rapport Notat-Sénard ${ }^{11}$ ), c'est-à-dire à minima, les salariés (incluant les intérimaires) et les actionnaires; il faut aussi considérer les responsabilités environnementales. Il faut aussi envisager qu'un entreprise puisse se définir une "mission» (ou une "raison d'être » comme le dit le rapport évoqué ) inscrite dans ses statuts. L'entreprise est donc pensée comme un projet de création collective qui engage les actionnaires. Et si il y a "mission», alors celle-ci est opposable aux actionnaires par les dirigeants et inversement. Cette mission devrait en outre qualifier et nommer les parties vis à vis desquelles l'entreprise souhaite s'engager et donc évaluer son action. La rédaction des statuts de la société stimule donc une discussion des parties constituantes, et des parties prenantes autour de ce qui est « visé » en commun. Notre équipe expérimente ce schéma de société à mission avec plusieurs partenaires souvent évoqués dans les grands médias : Nutriset, la CAMIF, CO cabinet de conseil pour l'économie sociale et solidaire,.. des start-ups aussi ont retenu ce modèle.... La mission ne se borne pas à quelques règles de mutualisation, mais essaie d'expliciter un mode de fonctionnement qui puisse être évalué. Nous recommandons l'instauration d'un conseil de mission à côté du Conseil d'administration. La mission peut prévoir une forme de codétermination avec les salariés, mais sans reprendre le modèle allemande. Mais la mission ne s'arrête pas aux frontières habituelles de

\footnotetext{
Et Aimée Moutet, Les logiques de l'entreprise, La rationalisation de l'industrie française dans l'entre deux guerres, Thèse soutenue en 1992 et publiée par les Editions de l'Ecole des Hautes études en sciences sociales en 1997.

10 Dans nos travaux avec Blanche Ségrestin et Kevin Levillain, nous avions parlé de parties « engagées » parce que ce qui fait « constitution » c'est l'engagement envers une autorité à qui la direction est confiée.

${ }^{11}$ Le rapport Notat-Sénard, « l'entreprise, objet d'intérêt collectif », publié le 9 mars, a été remis au gouvernement. Ses propositions sont proches de celles mis en avant par la recherche.
} 
l'entreprise quand il s'agit des énergies durables ou de la prévention du changement climatique.

Multitudes: La rénovation de l'entreprise semble prendre à l'étranger d'autres voies avec la B Corporation

Armand Hatchuel :

En effet, nous avons vu émerger aux Etats Unis dans les années 2000 un mouvement d' entrepreneurs qui voulaient travailler avec de nouvelles valeurs et ont défendu la création de nouvelles formes de société (Benefit corporations, Flexible purpose corporations..) . Mais ils ont eu beaucoup de difficultés, car aux Etats Unis, chaque Etat a son propre droit des sociétés, et la diffusion juridique devient très lente.

Les promoteurs de la benefit corporation ont aussi créé un label, attribué par un laboratoire privé sur la base d'une série de critères qu'il faut atteindre. Ce label revient à imposer une mission identique à toutes les B corps, ou en quelque sorte une Responsabilité Sociale et Environnementale minimale. Il nous semble que l'idée de label rigidifie la notion de mission et surtout risque de faire oublier l'importance politique, juridique et pédagogique d'un nouveau statut juridique. D’ailleurs, la Californie a créé la Flexible purpose corporation qui laisse chaque entreprise définir sa mission et surtout l'engagement de chacun pour la transition énergétique. C'est l'esprit que nous avons voulu transposé en droit français avec la Société à objet social étendu, la SOSE. ${ }^{12}$

Multitudes: les entreprises de l'économie sociale et solidaire semblent hostiles à l'institutionnalisation de l'entreprise à mission, pourquoi ?

Armand Hatchuel :

ils nous semblent qu'avec le rapport Notat Sénard cette position évolue ${ }^{13}$.Les entreprises de l'économie sociale et solidaire obéissent à des règles précises et se donnent des missions reconnues comme "sociales» du point de vue de l'Etat, voire d'utilité publique ou d'intérêt général, ce qui leur vaut des aides fiscales et en termes d'emploi. Les entreprises à mission ne leur sont pas concurrentes car elles peuvent se définir des règles et des objectifs qui ne correspondent pas à de tels engagements reconnus comme "sociaux » : objectifs scientifiques, territoriaux, de développement des compétences etc.. C'est le cas par exemple d'un orchestre d'handicapés qui en jouant partout veut contribuer à faire cesser la discrimination contre les handicapés là où on ne s'attend pas à la trouver. Les entreprises à mission sont plutôt des entreprises émergentes qui veulent se donner des garanties de pouvoir accomplir leur projet. Elles explorent un nouvel espace de l'action collective et du bien commun dont on ne peut a priori délimiter l'extension.

Propos recueillis par Anne Querrien

\footnotetext{
${ }^{12}$ Hatchuel A., Levillain K., Segrestin B. Vernac S., La Société à objet social étendu, Les Presses des Mines, 2015.

${ }^{13} \mathrm{Cf}$. le témoignage de la MAIF à la fondation Jean-Jaurès, en avril dernier.
} 